\title{
Intravitreal conbercept for branch retinal vein occlusion induced macular edema: one initial injection versus three monthly injections
}

\author{
X. Chen, T. M. Hu, J. Zuo, H. Wu, Z. H. Liu, Y. X. Zhan, Y. Xia, J. Wang and W. Wei
}

\begin{abstract}
Background: To compare the efficacy of one initial intravitreal injection of conbercept (IVC) versus three monthly IVCs in patients with macular edema (ME) after branch retinal vein occlusion (BRVO). Both options were followed by a pro re nata (PRN) retreatment regimen.

Methods: This study retrospectively investigated and followed 60 patients with acute ME secondary to BRVO for over a year. 30 subjects received one initial injection (1 + PRN group); while, 30 received three monthly injections (3+PRN group). The functional and anatomic outcomes were assessed during each follow-up.

Results: The general characteristics of the 60 subjects were as follows: mean [SD] age, 57.43 [13.06] years; 33 [55\%] female; 36 [60\%] non-ischemic form. Both groups showed a stable gain in visual acuity (VA) with similar logMAR (mean \pm SD) $(1+$ PRN group $0.308 \pm 0.399,3+$ PRN group $0.34 \pm 0.352)$ during the first 12 months. Additionally, both groups exhibited a significant reduction in central foveal thickness (CFT) with no statistically significant difference between them (1 + PRN group $222.1 \mu \mathrm{m} \pm 197.1 \mu \mathrm{m}, 3$ + PRN group $228.4 \mu \mathrm{m} \pm 200.2 \mu \mathrm{m}$ ). Both treatment groups had similar improvements in logMAR and anatomic outcomes over time. The stratified analysis showed that patients with the non-ischemic form and those with the ischemic form had similar improvements in VA $(0.346 \pm$ 0.366 VS $0.29 \pm 0.39, P=0.575)$ during the 12 months follow-ups. The number of injections was lower in the $1+P R N$ group (4.0 \pm 1.6$)$ than in the $3+$ PRN group $(4.7 \pm 1.3)(P=0.068)$. No adverse effects or unexpected safety issues were reported in either group.

Conclusions: Conbercept yielded significant improvements in VA and CFT among patients with BRVO induced ME, independent of their retinal ischemia status. The results showed that the $3+$ PRN regimen do not lead to better functional outcomes or lower treatment needs in clinical practice as compared to the $1+$ PRN regimen.
\end{abstract}

Keywords: Branch retinal vein occlusion, Macular edema, Conbercept, PRN

\footnotetext{
* Correspondence: 13951776603@163.com
}

Jiangsu Province Hospital of Chinese Medicine, Affiliated Hospital of Nanjing University of Chinese Medicine, Nanjing 210000, China

(c) The Author(s). 2020 Open Access This article is licensed under a Creative Commons Attribution 4.0 International License, which permits use, sharing, adaptation, distribution and reproduction in any medium or format, as long as you give appropriate credit to the original author(s) and the source, provide a link to the Creative Commons licence, and indicate if changes were made. The images or other third party material in this article are included in the article's Creative Commons licence, unless indicated otherwise in a credit line to the material. If material is not included in the article's Creative Commons licence and your intended use is not permitted by statutory regulation or exceeds the permitted use, you will need to obtain permission directly from the copyright holder. To view a copy of this licence, visit http://creativecommons.org/licenses/by/4.0/. The Creative Commons Public Domain Dedication waiver (http://creativecommons.org/publicdomain/zero/1.0/) applies to the data made available in this article, unless otherwise stated in a credit line to the data. 


\section{Background}

Retinal vein occlusion (RVO) is the second most common retinovascular disease after diabetic retinopathy, resulting in severe visual dysfunction among several patients worldwide [1]. A previously conducted pooled analysis showed that branch retinal vein occlusion (BRVO) $(0.44 \%)$ had a higher prevalence than central retinal vein occlusion (CRVO) (0.08\%) [2]. Clinically, observations of BRVO by fundus photography and fluorescein angiography are often indicative of retinovascular hemorrhage, dilated and tortuous retinal vessels, cottonwool spots, and retinal non-perfusion (NP).

Vascular endothelial growth factor (VEGF), through its complex interactions with vascular endothelial cell proliferation and vascular permeability, plays a key role in the pathogenesis of BRVO induced macular edema (ME) [3] and neovascularization [4, 5]. It has been well established that retinal nonperfusion and hemorrhages due to vascular occlusion can lead to an increase in VEGF production, hence exacerbating $\mathrm{ME}$ and ischemia [6]. In recent years, the use of anti-VEGF inhibitors has dramatically increased, making them the standard of care for BRVO-ME in many countries [7]. Studies have shown that intravitreal injections of anti-VEGF inhibitors are more effective in resolving $\mathrm{ME}$ and improving the best-corrected visual acuity (BCVA), including bevacizumab [8], ranibizumab [7], or aflibercept [9]. Conbercept (KH902; Chengdu Kanghong Biotech Co., Ltd., Sichuan, China) has been approved by the Chinese Food and Drug Administration for the treatment of RVO induced ME. It is a fusion protein that contains the extracellular domain 2 of VEGF receptor 1 and extracellular domains 3 and 4 of VEGF receptor 2 combined with the Fc portion of the human immunoglobulin G1. Previous studies have also shown that a regular pro re nata (PRN) use of VEGF blockade in RVO seems to have a significant and lasting effect on VA gain [10]. YUKO MIWA et al. [11] showed that there are no significant differences in functional outcomes between BRVO induced ME patients treated with a single monthly PRN injection of ranibizumab and those with three monthly PRN injections. The efficacy and safety of 3 monthly PRN injections of conbercept for the treatment of ME after RVO have been previously established by FALCON et al. [12]. Therefore, we evaluated the benefits and compared the visual and anatomical outcomes of a single monthly injection versus three monthly injections of conbercept in the treatment of ME after BRVO.

\section{Methods}

\section{Participants}

This retrospective study analyzed data from 60 patients with a confirmed diagnosis of macular edema after BRVO and a history of intravitreal injections of conbercept. The data in this study was collected from August 2017 to December 2019. All data originated from the Department of Ophthalmology, Jiangsu Provincial Hospital of Chinese Medicine (Nanjing, China). The purpose of the treatment and potential adverse effects were thoroughly explained, and all subjects signed informed consent before treatment initiation.

The eligibility criteria included the followings: patients should be at least 18 years of age; have decreased visual acuity; should have BRVO with retinal edema, and a central foveal thickness CFT $>250 \mu \mathrm{m}$ as assessed by optical coherence tomography (OCT). The exclusion criteria were: patients with their last intravitreal anti-VEGF or steroids treatment being within less than 6 months; patients with a history of ocular surgery; patients with an ocular disease such as diabetic retinopathy, senile cataract, and age-related macular degeneration, that significantly affected the BCVA; patients with a history of interventions or neovascularization (NV) before the study period; patients with advanced glaucoma, intraocular pressure $(\mathrm{IOP})>22 \mathrm{mmHg}$.

\section{Treatment}

The study sample collected devided into either the $1+$ PRN group or the $3+$ PRN group. 30 eyes received a single monthly intravitreal injection of conbercept, and 30 eyes received three monthly intravitreal injections of conbercept. An initial intravitreal conbercept injection was administered at day one, followed by a continuous monthly treatment until stabilization of BCVA. The retreatment criteria, according to the RPN scheme, were: vision loss of $\geq 10$ ETDRS letters compared with the previous month's BCVA; increase of ancentral subfield thickness $(\mathrm{CST}) \geq 50 \mu \mathrm{m}$; CST $>340 \mu \mathrm{m}$; presence of intraretinal fluid, intraretinal cysts, or subretinal fluid ME [13].

\section{Assessments}

The Early Treatment Diabetic Retinopathy Study (ETDRS) was used to evaluate temporal changes in best-corrected VA between the two groups. The secondary outcome was defined as pre and post-treatment mean changes in central retinal thickness (HD-OCT (Zeiss, Germany)) and is reflective of treatment response. The CFT was assessed by HD-OCT measurements between the vitreoretinal interface and the anterior boundary of the retinal pigment epithelium. Additional outcomes were defined as follows: the investigation and evaluation by fluorescein fundus angiography (FFA, Heidelberg, Germany) of ischemic or nonischemic BRVO and retinal circulation; the analysis and quantification of the number of injections during the 12 months follow-up. Safety assessments, including ocular, systemic adverse events (AEs), serious AEs (SAEs), and their correlation with the treatment, as well as injection procedures, were carried out at each follow-up. 


\section{Statistical analysis}

All statistical analyses were carried out using SPSS 20.0 (SPSS, Inc., Chicago, IL, USA). Quantitative data were presented as mean $\pm \mathrm{SD}$. VA measurements were converted to a logarithm of the minimum angle of resolution ( $\log M A R)$ for statistical analysis. A paired t-test was used to analyze changes in VA as well as CFT within each group. Meanwhile, an unpaired t-test was used to compare the two parametric data between treatment groups. Differences in distributions were analyzed through chi-square tests. A $P$ value $<0.05$ was considered statistically significant.

\section{Results}

\section{Study population and baseline characteristics}

Among the 60 participants in this study, 30 (50\%) received a single initial IVC, and $30(50 \%)$ received three monthly IVC. The baseline characteristics of each study group are summarized in Table 1 . There were similarities in patient demographics and baseline ocular characteristics between the two groups. 36 eyes were classified as non-ischemic, and 24 eyes were classified as ischemic after FFA. All patients in this study are Asian.

\section{Change in best-corrected visual acuity and central foveal thickness}

Both groups showed significant improvement in mean BCVA and reduction in mean CFT one month after the initiation of the treatment. Figure 1 shows significant improvements in overall mean BCVA $(0.324 \pm 0.373)$ from the baseline values to the 12 months follow-up in all treated eyes $(P<0.001)$. The 12 months follow-up indicated a significant reduction in overall mean CFT $(225.3 \mu \mathrm{m} \pm 197 \mu \mathrm{m})$ of all treated eyes $(P<0.001)$. Figure 2 indicates an increase in means BCVA $(0.308 \pm 0.399$ logMAR and $0.34 \pm 0.352 \log$ MAR) at 12 months in the $1+$
PRN and the $3+$ PRN groups, respectively. No statistically significant difference was seen between the two groups $(p=0.741)$. Both groups showed a significant decrease in means CFT $\quad(222.1 \mu \mathrm{m} \pm 197.1 \mu \mathrm{m} \quad$ and $228.4 \mu \mathrm{m} \pm$ $200.2 \mu \mathrm{m})$ at the 12 months follow-up, with no statistically significant difference between them $(p=0.902)$. A secondary analysis indicated that CFT tended to be smaller and VA to be better in the $3+$ PRN group compared to the $1+$ PRN group in the 12 months follow-up. Additionally, the analysis indicated that the choice of the treatment regimen was no association with the final BCVA and final CFT $(P>0.05)$.

\section{Stratified analysis of best-corrected visual acuity and central Foveal thickness}

We stratified patients according to baseline FFA examinations. Patients with a peripheral capillary nonperfusion greater than five disc areas were categorized as ischemic BRVO ( $n=24$ eyes), and the remaining were classified as non-ischemic BRVO ( $n=36$ eyes). Figure 3 shows the mean CFT and the logMAR VA in each subgroup. The findings indicated significant improvements in VA $(0.346 \pm 0.366 \log$ MAR in the non-ischemic and $0.29 \pm 0.39 \log$ MAR in the ischemic subgroups) at the 12 months follow-up. However, no statistically significant difference was observed between the two subgroups $(P=0.575)$. Even so, the non-ischemic subgroup exhibited better mean VA than the ischemic subgroup during the entire observation period. Similarly, a significant decrease in CFT $(225.5 \mu \mathrm{m} \pm 219.8 \mu \mathrm{m}$ in the non-ischemic subgroup and $225 \mu \mathrm{m} \pm 161.3 \mu \mathrm{m}$ in the ischemic subgroup) was seen at the 12 months follow-up. Nevertheless, there was no statistically significant difference between the two subgroups $(P=0.993)$.

Table 1 Baseline Characteristics of the Study Population

\begin{tabular}{|c|c|c|c|c|}
\hline & Total & $1+$ PRN Group & $3+P R N$ Group & $P$ \\
\hline \multicolumn{5}{|l|}{ Baseline } \\
\hline Number (patients/eyes) & $60 / 60$ & $30 / 30$ & $30 / 30$ & NA \\
\hline Gender(Male/ Female) & $27 / 33$ & $15 / 15$ & $12 / 18$ & 0.436 \\
\hline Age, years & $57.43 \pm 13.06$ & $57.33 \pm 14.47$ & $57.53 \pm 11.73$ & 0.953 \\
\hline Diagnosis (non-ischemic/ ischemic) & $36 / 24$ & $16 / 14$ & 20/10 & 0.292 \\
\hline BCVA (LogMAR) & $0.758 \pm 0.413$ & $0.77 \pm 0.371$ & $0.746 \pm 0.466$ & 0.823 \\
\hline $\mathrm{CFT}, \mu \mathrm{m}$ & $520.4 \pm 186.5$ & $522.4 \pm 191.5$ & $518.4 \pm 184.5$ & 0.935 \\
\hline \multicolumn{5}{|l|}{ Final } \\
\hline BCVA (LogMAR) & $0.434 \pm 0.283$ & $0.462 \pm 0.261$ & $0.405 \pm 0.306$ & 0.445 \\
\hline CFT, $\mu \mathrm{m}$ & $295.1 \pm 71.2$ & $300.2 \pm 89.3$ & $290 \pm 47.7$ & 0.580 \\
\hline Number of injections during $12 \mathrm{mo}$ & $4.4 \pm 1.5$ & $4.0 \pm 1.6$ & $4.7 \pm 1.3$ & 0.068 \\
\hline
\end{tabular}

PRN pro re nata, BCVA best-corrected visual acuity, LogMAR the logarithm of the minimum angle of resolution, CFT Central foveal thickness Continuous variables were presented as mean and SD

$P$-value $<0.05$ was considered statistically significant 


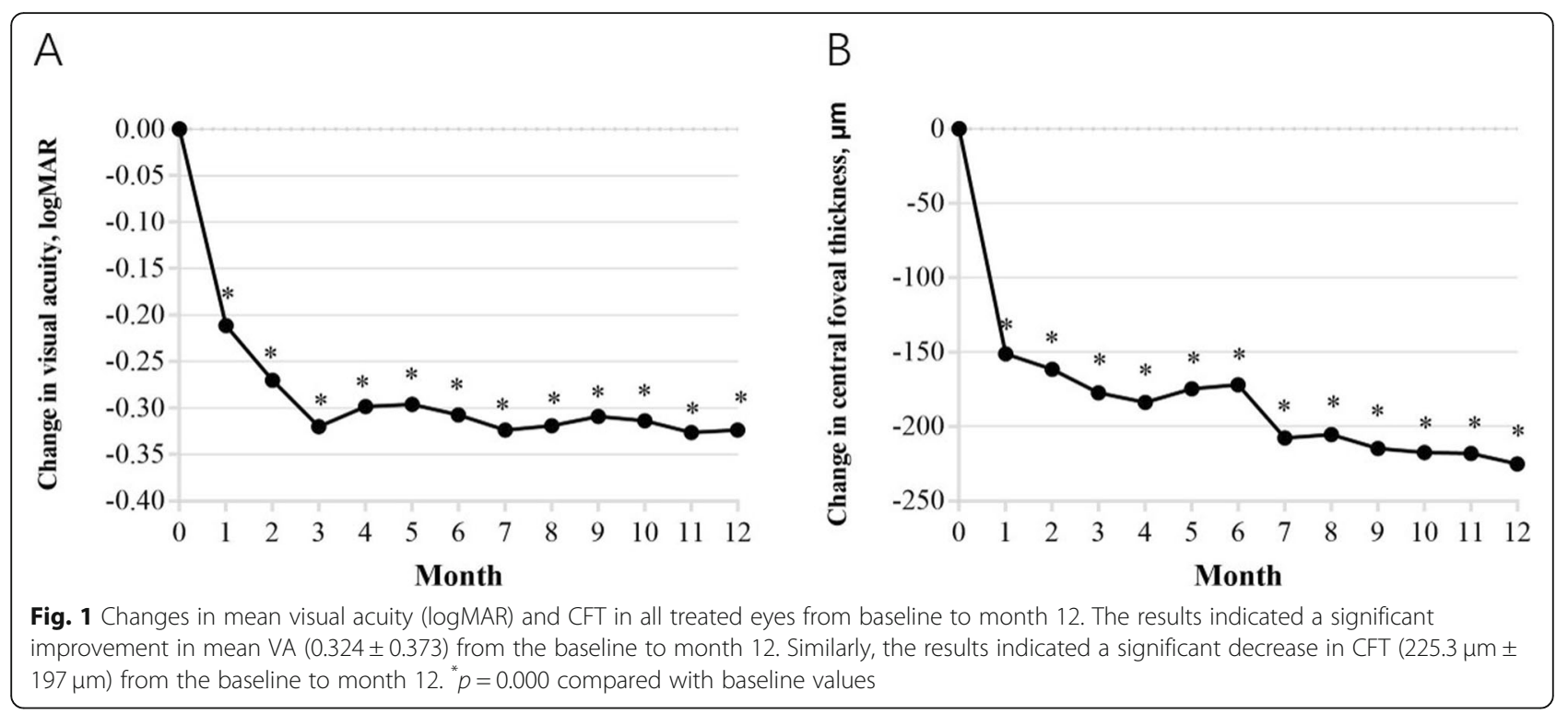

\section{Number of pro re nata Conbercept injections}

Over the 12 postoperative months, the mean \pm SD number of conbercept injections in patients receiving the $1+$ PRN and the $3+$ PRN (range 2-9) were $4.0 \pm 1.6$ and $4.7 \pm 1.3$, respectively. There was no significant difference between the two groups $(P=0.068$, Table 1$)$. The total number of PRN injections was significantly associated with a shorter duration until the first PRN injection in both groups $(R=-$ $0.459, P=0.006, R=-0.6, P=0.000$, respectively, Fig. $4 \mathrm{~b}$ ).

\section{Safety assessments}

Adverse events of interest were rare during the entire observation period, and all patients in the study did not show significant side effects.

\section{Discussion}

Although the traditional PRN treatment regimens have demonstrated their usefulness in the treatment of BRVO, the debate of whether their efficacy outways the resultant patient burden on the health care system remains. In recent years, anti-VEGF intravitreal therapy has been extensively used for the treatment of ME associated with BRVO. This study was conducted to assess the effects of monthly intraocular injections of conbercept through a span of 12 months. According to our results, even though the overall mean number of injections was $4.4 \pm 1.5$, the use of conbercept with PRN regimens yielded favorable outcomes in visual acuity and steady reductions in $\mathrm{ME}$.

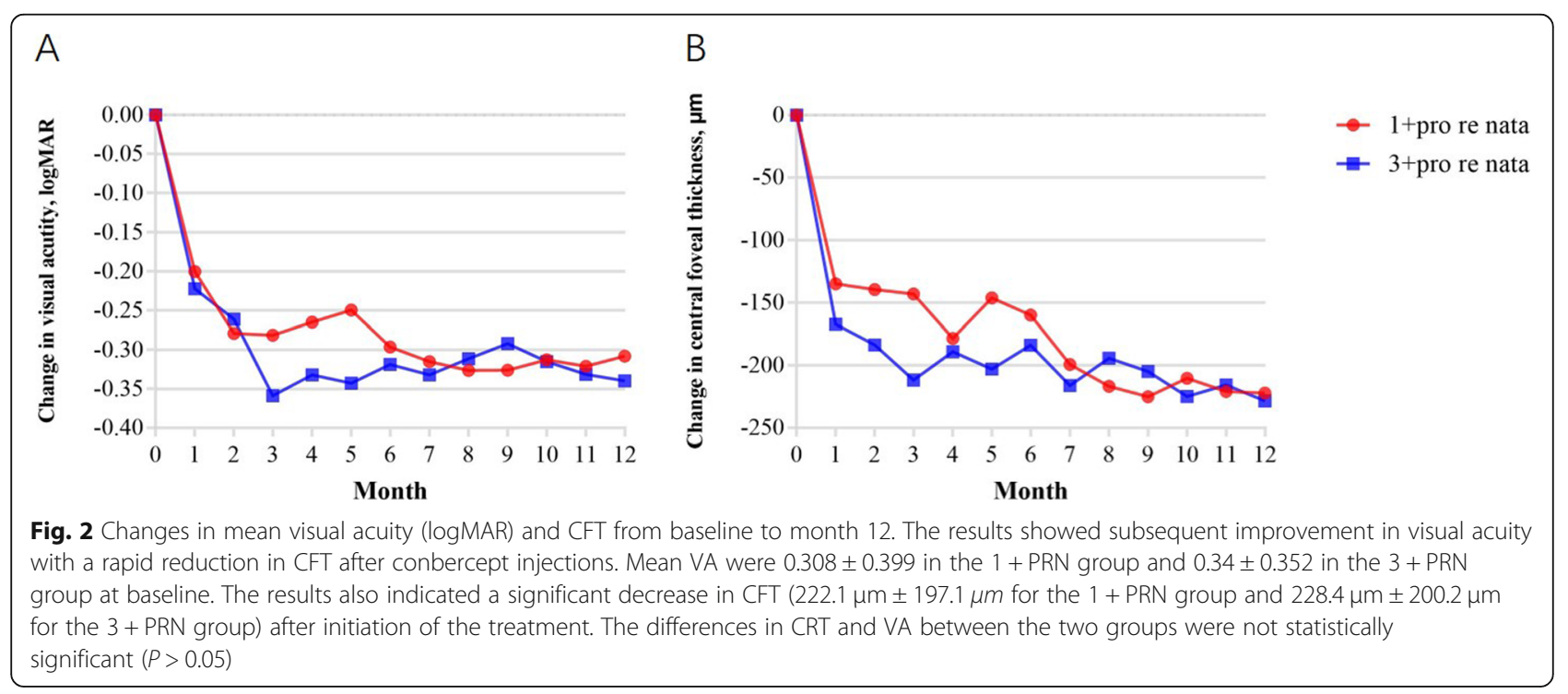




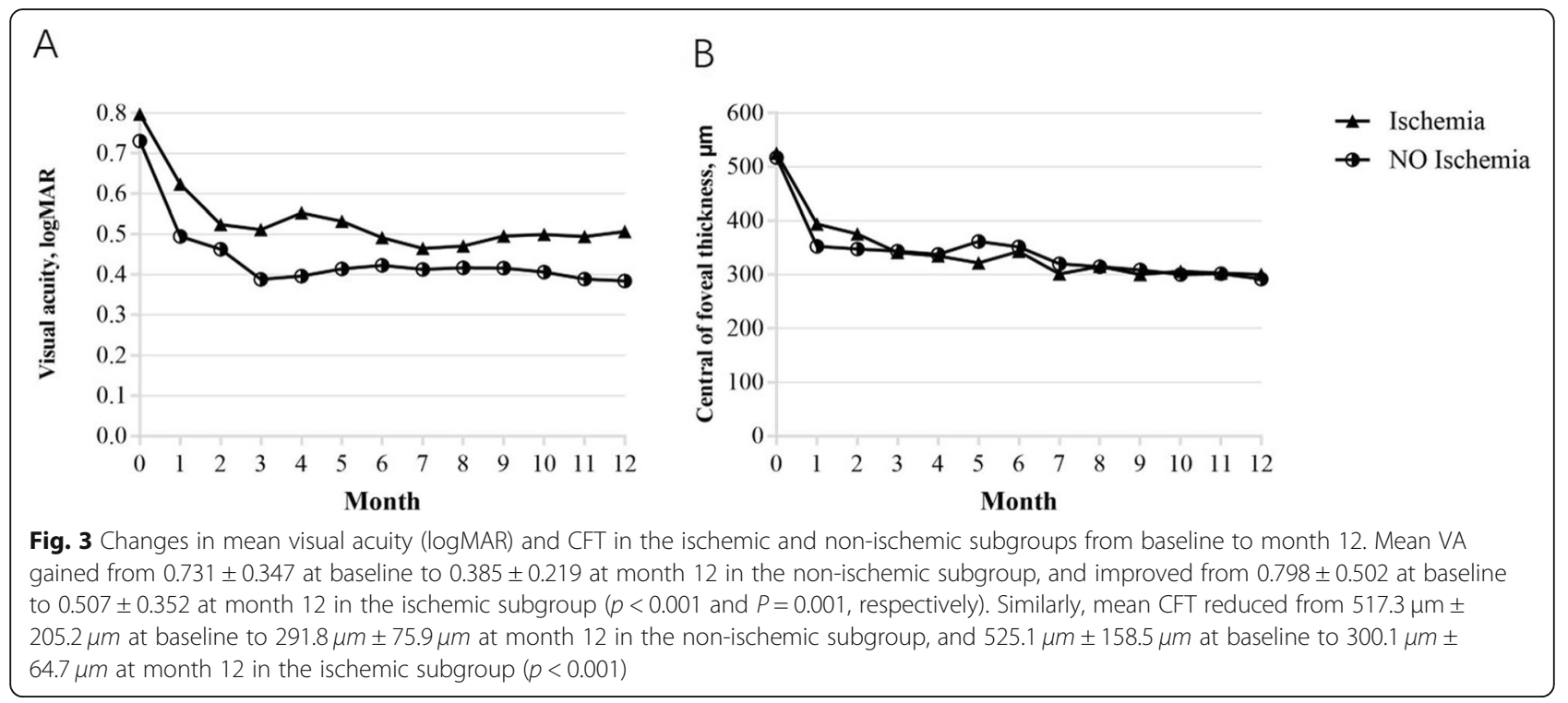

The overall sample is comprised of 60 eyes, with 30 eyes allocated to the $1+\mathrm{PRN}$ group and 30 eyes to the $3+$ PRN group. The results showed that VA and CFT at baseline were not significantly different between the two treatment groups. Further analysis indicated that the $1+$ PRN group showed significant improvement in mean logMAR VA (from $0.77 \pm 0.371$ at baseline to $0.462 \pm$ 0.261 at month $12(p<0.001))$ at 12 months follow-up. Similarly, the $3+$ PRN group also exhibited improvements in mean $\log$ MAR VA (from $0.746 \pm 0.466$ at baseline to $0.405 \pm 0.306$ at month $12(p<0.001))$ during the last follow-up. Additionally, both groups showed significant decrease in mean CFT (from $522.4 \mu \mathrm{m} \pm 191.5 \mu \mathrm{m}$ at baseline to $300.2 \mu \mathrm{m} \pm 89.3 \mu \mathrm{m}$ at month 12 (p< 0.001 ) for the $1+\mathrm{PRN}$ group and from $518.4 \mu \mathrm{m} \pm$ $184.5 \mu \mathrm{m}$ at baseline to $290 \mu \mathrm{m} \pm 47.7 \mu \mathrm{m}$ at month 12
( $p<0.001)$ for the $3+\mathrm{PRN}$ group, respectively) during the last follow-up. There was no significant difference in the final mean VA or final CFT between the two regimens. The total number of injections per eye in the $1+$ PRN group (4.0 \pm 1.6$)$ was slightly lower than that in the $3+$ PRN group $(4.7 \pm 1.3)(P=0.068)$. A reason for this may be that conbercept displayed long half-life and strong bioavailability [14]. $1+$ PRN regimen could support a prolonged dosing interval than $3+\mathrm{PRN}$ regimen in disease stability criteria. However, patients tended to have greater CFT and worse VA in the $1+$ PRN group as compared to the $3+$ PRN group during the observation period (although the changes of VA and CFT were not statistically significant between the two groups). Moreover, lower fluctuation of mean logMAR VA and CFT were observed in patients belonging to the $3+$ PRN

\section{A}

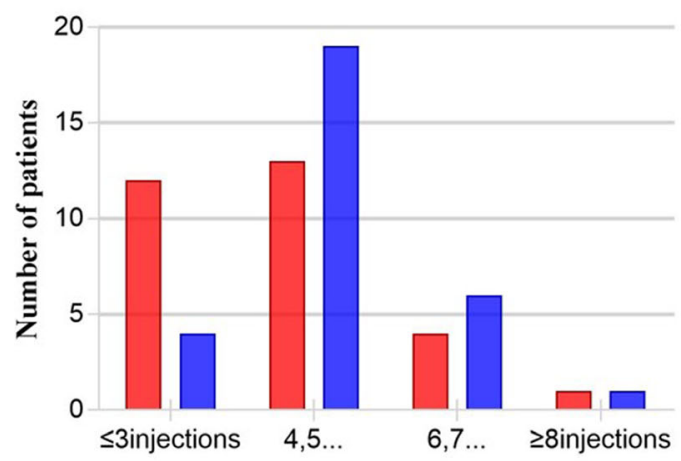

Number of injections in both groups
B

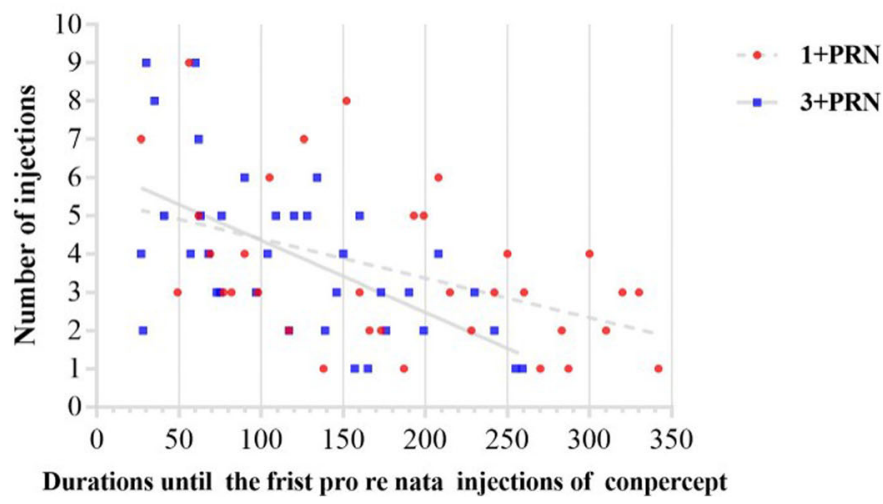

Fig. 4 B. Scatter plots showing the association between the number of PRN conbercept injections and the duration until the first PRN injection. The total number of PRN injections was significantly associated with shorter durations until initial PRN injection in both groups $(R=-0.459, P=$ $0.006, R=-0.6, P=0.000$, respectively) 
group at the 12 months follow-up. ZUHUA SUN et al. [12] previously reported that the differences in mean VA values between $B R V O$ and CRVO groups were relatively small $(17.83 \pm 10.89$ letters VS $14.23 \pm 11.74)$, indicating that IVC is generally safe and effective. Thus, taking into account our findings, it is clear that the reductions in mean CFT and gains in mean VA in the $1+$ PRN regimen are almost similar to those of the $3+$ PRN regimen. A recent study [15] with similar study designs and using PRN treatment protocols also had similar outcomes. Both regimens showed similar efficacy.

Our study not only included patients with PRN treatment regimens but also those with non-ischemic or ischemic BRVO induced ME. We observed higher injection numbers on average in eyes belonging to the ischemic subgroup $(4.6 \pm 1.7)$ as compared to those in the non-ischemic subgroup $(4.2 \pm 1.3, P=0.308)$. The baseline data showed that compared to the 36 nonischemic eyes, the 24 eyes in the ischemic subgroup had slightly worse CFT and VA values. However, the difference was not statistically significant. This may be partially due to the correlation between ischemia and greater CFT, as well as poorer VA [16]. Stratified analysis of the non-ischemic subgroup showed a rapid reduction in ME and significant improvement of BCVA at month 12 (from $0.731 \pm 0.347$ at baseline to $0.385 \pm$ $0.219, p<0.001)$. In contrast, the analysis of the ischemic subgroup indicated a reduction in ME with slow improvement in VA at month 12 (from $0.798 \pm 0.502$ at baseline to $0.507 \pm 0.352, P=0.001$ ). Additionally, VA tended to be lower in the ischemic subgroup as compared to the non-ischemic one. Consequently, the findings suggested that these factors are associated with the visual functional outcome.

It is worth noting that the novelty of this study is that it provides crucial information on anti-VEGF therapy and shows that it can achieve a promising outcome and significantly improve VA and CFT regardless of the nature of the ME (non-ischemic or ischemic). In this study, 10 of the ischemic subgroup patients received conbercept +laser treatments. Interestingly, The BRIGHTER study has previously shown that the addition of laser does not lead to better functional outcomes or lower treatment needs [17]. In their study, Ramin Tadayoni et al. compared changes in BCVA outcome and ranibizumab injections between the ranibizumab + laser and the ranibizumab monotherapy groups. They concluded that there were no significant differences in VA and numbers of ranibizumab injections between groups (11.3 vs. 11.4). Another recent clinical trial [18] demonstrated that the trend of improvement in VA and reduction of CMT observed during conbercept usage for the treatment of ME secondary to BRVO were similar to those obtained with ranibizumab.
In summary, our study reported findings on the efficacy of anti-VEGF treatment and provided additional data on PRN protocols in patients with BRVO-ME. It showed that there were no significant differences in outcomes between the $1+$ PRN and the $3+$ PRN groups. Additionally, visual stabilization was observed after a prolonged treatment period and regular anti-VEGF dosing, hinting that the adoption of such protocol might help reduce the treatment burden. One of the major limitations of this study is its small number of participants and its relatively short follow-up period. PRN regimens are likely to be beneficial for patients with such conditions. However, future research should focus on larger controlled studies and long-term dosing strategies to confirm our data.

\section{Conclusions}

This observational study confirms that Conbercept resulted in clinically and statistically significant visual and anatomic benefits for patients with ME secondary to BRVO. Three monthly injections might be more appropriate with patients even though no significant differences between the $1+$ PRN and the $3+$ PRN groups.

\section{Abbreviations \\ BRVO: Branch retinal vein occlusion; IVC: Intravitreal injection of conbercept; ME: Macular edema; PRN: Pro re nata; BCVA: Best-corrected visual acuity; logMAR: Logarithm of minimum angle of resolution; CFT: Central foveal thickness; OCT: Optical coherence tomography; FFA: Fluorescein fundus angiography; ETDRS: The Early Treatment Diabetic Retinopathy Study; SD: Standard deviation}

\section{Acknowledgments}

Not applicable.

\section{Authors' contributions}

$X C$ and WW designed and analyzed the study. TMH, ZHL and YXZ carried out the measurements. $X C$ wrote the manuscript with support from WW and JZ. JW, YX and HW helped interpretation of data. All authors have read and approved the manuscript.

\section{Funding}

This work was supported by grants from the National Natural Science Foundation of China (81774370). The funding body participated in the design of the study, analysis and interpretation of data and in writing the manuscript.

\section{Availability of data and materials}

The datasets generated and/or analysed during the current study are not publicly available due to the prevision of further publications coming soon, but are available from the corresponding author on reasonable request.

\section{Ethics approval and consent to participate}

All performed procedures in this study were done according to the ethical standard of The Jiangsu Province Hospital of Chinese Medicine and adhered to the 1964 Helsinki declaration and its latest amendments.

\section{Consent for publication}

Not applicable.

Competing interests

The authors declare that they have no competing interests. 
Received: 30 March 2020 Accepted: 1 June 2020

Published online: 11 June 2020

\section{References}

1. Tah V, Orlans HO, Hyer J, et al. Anti-VEGF therapy and the retina: an update. J Ophthalmol. 2015;2015:627674.

2. Rogers $\mathrm{S}, \mathrm{Mclntosh} \mathrm{RL}$, Cheung $\mathrm{N}$, et al. The prevalence of retinal vein occlusion: pooled data from population studies from the United States, Europe, Asia, and Australia. Ophthalmology. 2010;117(2):313-19.

3. Campochiaro PA, Brown DM, Awh CC, et al. Sustained benefits from ranibizumab for macular edema following central retinal vein occlusion: twelve-month outcomes of a phase III study. Ophthalmology. 2011;118(10): 2041-9.

4. Vance SK, Chang LK, Imamura Y, et al. Effects of intravitreal anti-vascular endothelial growth factor treatment on retinal vasculature in retinal vein occlusion as determined by ultra wide-field fluorescein angiography. Retin Cases Brief Rep. 2011;5(4):343-7..

5. PA C. Anti-vascular endothelial growth factor treatment for retinal vein occlusions. Ophthalmologica. Journal international d'ophtalmologie. International journal of ophthalmology. Zeitschrift fur Augenheilkunde. 2012:30-5.

6. Koss MJ, Pfister M, Rothweiler F, et al. Comparison of cytokine levels from undiluted vitreous of untreated patients with retinal vein occlusion. Acta Ophthalmol. 2012;90(2):e98-e103.

7. Campochiaro PA, Heier JS, Feiner L, et al. Ranibizumab for macular edema following branch retinal vein occlusion: six-month primary end point results of a phase III study. Ophthalmology. 2010;117(6):1102-12 e1..

8. Leitritz MA, Gelisken F, Ziemssen F, et al. Grid laser photocoagulation for macular oedema due to branch retinal vein occlusion in the age of bevacizumab? Results of a prospective study with crossover design. $\mathrm{Br} \mathrm{J}$ Ophthalmol. 2013;97(2):215-9.

9. Campochiaro PA, Clark WL, Boyer DS, et al. Intravitreal aflibercept for macular edema following branch retinal vein occlusion: the 24-week results of the VIBRANT study. Ophthalmology. 2015;122(3):538-44..

10. Wecker T, Ehlken C, Bühler A, et al. Five-year visual acuity outcomes and injection patterns in patients with pro-re-nata treatments for AMD, DME, RVO and myopic CNV. Br J Ophthalmol. 2017:101(3):353-9.

11. Miwa $Y$, Muraoka $Y$, Osaka R, et al. Ranibizumab for macular edema after branch retinal vein occlusion: One initial injection versus three monthly injections. Retina (Philadelphia, Pa). 2017;37(4):702-9.

12. Sun Z, Zhou H, Lin B, et al. Efficacy and safety of intravitreal conbercept injections in macular edema secondary to retinal vein occlusion. Retina (Philadelphia, Pa.). 2017;37(9):1723-30..

13. Willoughby AS, Vuong VS, Cunefare D, et al. Choroidal Changes After Suprachoroidal Injection of Triamcinolone Acetonide in Eyes With Macular Edema Secondary to Retinal Vein Occlusion. Am J Ophthalmol. 2018;186: 144-51..

14. Liu K, Song Y, Xu G, et al. Conbercept for treatment of Neovascular agerelated macular degeneration: results of the randomized phase 3 PHOENIX study. Am J Ophthalmol. 2019;197:156-67.

15. Osaka R, Muraoka Y, Miwa Y, et al. Anti-Vascular Endothelial Growth Factor Therapy for Macular Edema following Central Retinal Vein Occlusion: 1 Initial Injection versus 3 Monthly Injections. Ophthalmologica. 2018;239(1): 27-35..

16. Singer $M$, Tan CS, Bell D, et al. Area of peripheral retinal nonperfusion and treatment response in branch and central retinal vein occlusion. Retina (Philadelphia, Pa.). 2014;34(9):1736-42.

17. Tadayoni R, Waldstein SM, Boscia F, et al. Sustained benefits of Ranibizumab with or without laser in branch retinal vein occlusion: 24-month results of the BRIGHTER study. Ophthalmology. 2017;124(12):1778-87.

18. Li F, Sun M, Guo J, et al. Comparison of Conbercept with Ranibizumab for the treatment of macular edema secondary to branch retinal vein occlusion. Curr Eye Res. 2017;42(8):1174-8.

\section{Publisher's Note}

Springer Nature remains neutral with regard to jurisdictional claims in published maps and institutional affiliations.

\section{Ready to submit your research? Choose BMC and benefit from:}

- fast, convenient online submission

- thorough peer review by experienced researchers in your field

- rapid publication on acceptance

- support for research data, including large and complex data types

- gold Open Access which fosters wider collaboration and increased citations

- maximum visibility for your research: over $100 \mathrm{M}$ website views per year

At BMC, research is always in progress.

Learn more biomedcentral.com/submissions 